\title{
Model predictive control of magnetic levitation system
}

\author{
Lafta E. Jumaa Alkurawy ${ }^{1}$, Khalid G. Mohammed ${ }^{2}$ \\ ${ }^{1}$ Department of Electronics Engineering, Universitas of Diyala, Iraq \\ ${ }^{2}$ Department of Electrical Power and Machine Engineering, Universitas of Diyala, Iraq
}

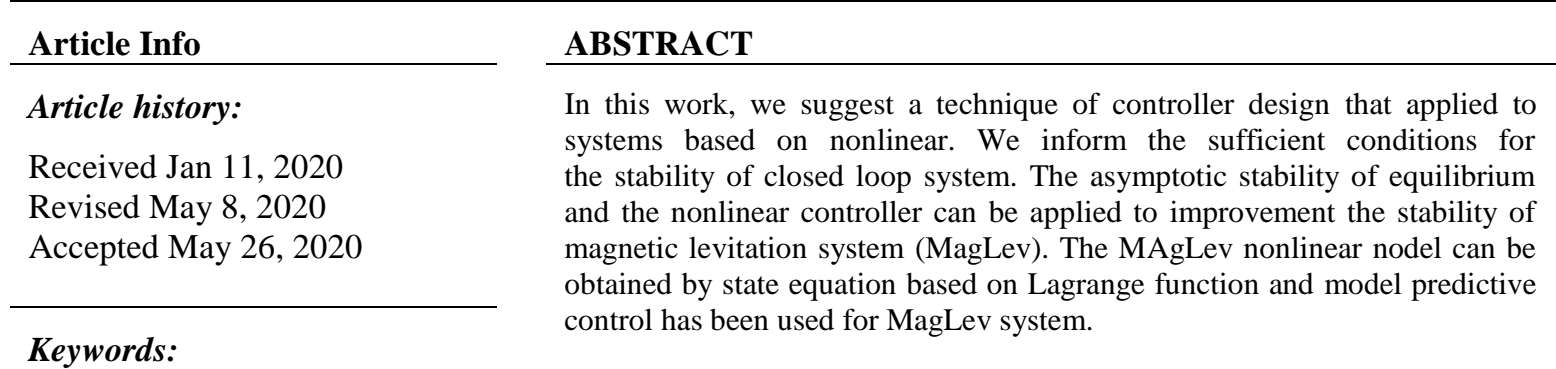

Machine

Magnetic levitation system

Modeling of nonlinear

MPC control

\section{Corresponding Author:}

Lafta Esmaeel Jumaa Alkurawy,

Department of Electronics Engineering,

University of Diyala,

Quds Square, Baquba, Diyala, Iraq

Email: lafta_67@yahoo.com

\section{INTRODUCTION}

The main problem of the magnetic levitation system (MagLev) is human that causes the death is a disease of heart. The diseases have different form, from the simplest to difficult and risks forms as transplantation of heart. Since the technology upgrade, it is feasible to get new chances in medical tools to inform the risks to our heart. There are many modeling of heart of human have been applied by physical model and compare with real systems.

Seo, et al., [1], they proposed the results of experimental and analysis of a single- sided for motor which is a linear induction (SLIM) normal and thrust for magnetic levitation (maglev) trans which is semi high speed. Takao, et al., [2], they studied a magnetic levitation system (Maglev) with ferromagnetic material and high temperature superconducting (HTS). They improved the properties of levitation system by changing the permanent magnet into a high temperature superconducting. The results of numerical analysis displayed that the force of maximum restoring and force of maximum levitation were $338 \mathrm{~g}$ and 1550 , respectively, which are 4.6 and 14 times greater than the parameters for a system that based on permanent magnet. They displayed their data as the force of peak levitation decreased, the distance increased, while the force of resorting increased. They improved and succeeded the properties of levitation system.

Sun, et al., [3], they developed a vehicle of hybrid magnetic levitation system utilizing superconducting maglev (SML) and permanent maglev system (PML). It is collected of a maglev frame, an SML and a PML. The frame of maglev is structured to help the load body of vehicle and associated the components of maglev. The part of SML with forces of pinning is used to assurance the force, the part of PML with disharmony force is structured to help the load. A measurements of vibration acceleration for the composite vehicle was organized by dissimilar states, incorporating different different pulse and loads irritations. The products of experimental display the features of dynamic are dissimilar between the lateral 
and vertical orientations. They got results to help an insight to the vehicle of composite magnetic levitation employed PML, SML and establish argument of the optimization and promotion

Lim, et al., [4], they presented the characteristics of electromagnetic for an induction motor in nonlinear properties for MagLev in high speed. The magnetic levitation train impetus system supplies exceptional ride standard, but has drawback in the aptitude of propulsion is little owing to its intrinsic properties. The maglev trains impetus system with comparatively huge air aperture, the expand in the efficiency of propulsion stays a significant problem. The impetus system makes an upright force through impetus control system. The properties of electromagnetic for the vertical force of impetus system to be accurate the stability of the impetus system.

Chen, et al., [5], they studied a technique to examine the features of the electromagnetic for the MagLev which comprises permanent magnets (PMs) and high temperature superconductor (HTS). The method is constructed of finite element incorporating with $\mathrm{H}$ infinity formulation, that is useful for axis in 2-D symmetric issues which comprise the material of high temperature superconductor. The force of axial levitation for the model is calculated properties of nonlinear for HTS bulk. The results of experiment of the system are acquired and contrasted with results of simulation with the results that show between simulation and experimental are matched well.

$\mathrm{Xu}$, et al., [6], they suggested a real-time wrench model (RRWM) with FPGA; for the moment an MLA able of rotation and translation in the horizontal plane. In order to get the high precision with perfect mostly; the distribution of torque and force is worked out by node of magnetic. The calculation components are expanded on (FPGA) by utilizing the parallel architecture processing and pipelined arithmetic. In the test, the RTWM of the computational accuracy and suggested MLA is contrasted with the method of finite element. The results display that the divergence of relative is not more than $2 \%$ selecting the results of FEM as a point of reference.

Chen, et al., [7], they suggested a novel of magnetic levitation (Maglev) system for 6-DOF to progress the design and robust. The concept of design endeavors to keep excellent performance in trip of moving instead than position to position for accuracy. They attempted to expand the system with range of large predicted. They structured the force model in relating dissimilarity from the distance to forces of magnetic levitation and obviate the restriction of maglev to be repulsive levitation. They adopted and built the system and matched the experimental results.

Spałek [8], he proposed the solution of analytical method for maglev problem for conductive balls and magnetically anisotropic. The magnetic field uses swirl currents in the conductive balls and magnetic. The force of material by inhomogeneity of reluctivity and the force by swirl current lead into levitation ball. The maglev field is regulated by manners of method of variable separation. He calculated the forces of material acting on the ball and Lorentz and calculated the power loses by manners of Poynying surface integral and Joule volume integral and used Maxwell to evaluate the total force.

Morandi, et al., [9], they investigated the force of levitation is between a permanent magnet and yttrium barium copper oxide (YBCO) which it is single-grin. The force tested in condition of field cooling and cooling with zero field. They analyzed the power dissipation and peak levitation force and used a numerical analysis to follow up the electrodynamics of maglev system. They obtained a pertinent observed for cycle of levitation at temperature with high degree and a good production for the measured data. They obtained critical current density by manners of the numerical model to fir the measured data.

Chen, et al., [10], they studied and improved the seal capacity and sealing life for maglev system. They used the finite element method to predict the magnetic field. The magnetic fluid thickness on the reciprocating field surface and the magnetic fluid flow rate are got by theatrical method; the capacity of static seal is related to the volume of fluid by measurements results. The capacity of static seal got by experiment is nearly equivalent to the simulation value with the fluid of the magnetic. They got a good result between the motion of shaft and sealing life and it decreases with increasing the velocity and stroke.

Qiy, et al., [11], they proposed a novel design for controller technique which the system is nonlinear. They established some adequate conditions that are capable for stability with closed loop by methodology of classical power shaping. By Lyapunov theory, they did the controller with nonlinear syatem and the asymptote of required equilibria stability. They designed a performance controller to prove the maglev system methodology.

Lahdo, et al., [12], they proposed the analytical calculation of maglev system is alternative to related to time with three dimensions by finite element technique due to low computational cost and higher accuracy. They determined the levitation force of magnetic guidance by analytical method that has a significant tool for high precision maglev system and controlling. The important result is the position dependence of the force of magnetic levitation over the range of whole travel.

Venghi, et al., [13], they developed a maglev control system. They linearized the non-linear system model around the operating point. The system has two control loops, an outer position loop and an inner 
current loop are structured by using the linear system. Experimental and simulation results are being seen to verify the dynamic response of suggested control.

Sivrioglu, et al., [14], they studied the concept of multi surface levitation by using one high temperature superconductor (HTS) and three permanent magnets (PMs) in a levitation configuration to get better stiffness in a superconducting magnetic bearing system. They measured the realized and levitation forces by an experiment and the levitation surfaces are attractive polarization of the PMs with the large levitation force.

Patel, et al., [15], they interested for applications of maglev because of the geometry pliability for permitting them to be made huge narrow slabs comfortable for linear magnetic levitation system bearings and planar rotary magnetic bearings. They measured the forcwe of axial levitation force was between a $25 \mathrm{~mm}$ diameter of permanent magnetic and slab of field cooled with $30 \mathrm{~mm}$ values which gave a field necessary of cylindrically symmetric of rotory bearings. They got a good result for permanent magnet and bigger $43 \mathrm{~mm}$.

Amrhein, et al., [16], they proposed to design a technology for magnetic bearing complex in applications with volumes of higher production. They presented some stimuli and ideas because on measures with low- cost in this area of field is scarce and these ideas are (i) motor with bearingless flux-switching, (ii) motor of a brushless permanent manager with additional axial bearing capability, and (iii) bearing of highly integrated hybrid that sipplies the degree of freedom with active stabilization. They described the particular of operation features and designs, and contrast the additional the costs of electrical and mechanical of these with technologies of standard motor and they got a good analysis with the stability.

Abdulwahhab [17], he proposed to design a controller of adptive state feedback for magnetic levitation system which is nonlinear and unstable system. They achieved settling time of 1 sec and maximum overshoot $1.5 \%$ by method of pole-placement is done by linerization the nonadaptive state feedback. The designed controller may no longer active when the operating point changes, because the designed focused on a linearization about diferent opearating point. The simulation results show that the operating point has notable impact on execution of nonadaptive state feedback control.

Quang, et al., [18], the design a motor of polysolemoid linear (PLM) in many aspects of material due to its operations, in which the motion is straight that it is provided directly witout actuators of mediate mechanical. Some methods of control for PLM focused on motor of rotation have been applied with commons on model by mathematic manner, but position, constraints of current and velocity which are significant in real syatem have been neglected. They used model predictive control to control the control of position tracking problem for PLM. The controller for a system forces the position errors of tracking to small region of satisfies state and original of constraints including current, position and velocity.

Quang, et al., [19], they designed a control for the tracking of velocity and position to guarantee boundaries of voltage control and its variation. A strategy of cascade control involving a loop of inner and outer loop is applied to synchronous linear motor. The model predictive control (MPC) is used with Laguerre model for inner loop and outer controller was designed for method of nonlinear damping and they got a validate performance of the controller with numerical simulation.

Okokpujie, et al., [20], they determined the techniques of active queue management robustness to varying load, propagation delay in a wireless environment, and link capacity. The transmission control protocol queue management is contrlloled by four stanjdard were compared. The controller of active queue management was the model predictive control (MPC), self-tuning regulator (STR), randon early detection and fixed-parameter proportion integral (PI). The algorithm of congestion control robustness of each technique was calculated by varying the condictions in simulating by Simulink software in MATLAB. The controller by MPC gives the good results in controllability in wireless network and response time with varying propagation delay and link capacity.

Firdose and Rao [21], they introduced a predictive optimization of risk management model with software engineering. The PROM important contribution is display credible calculation of risk analysis by develoopement of information technolohy (IT) with software practices. The designing of PROM with measurement of risk factor is approach of machine learning without participation of iteration. Outcome of this stusy displys that system of PROM proposals mathematically cost influential of risk elements as evaluated to standards of quality for system included in projects with software method.

Chandriah and Raghavendra [22], they proposed an analytical model with optimization of predictive is implemented with regard to span the hole between damands and supply in supply chain 4.0. The framework of anaylytic is a manufacture from derived of constraints from the environment of practical to display the best pertinence of it. Outcome of this study displays the suggested model could show the best performance in correspondence to the method of optimization with the best of control of budget for system.

Ikaouassen, et al., [23], they investigated to improve the strategy of curren predictive model control (CPMC) with one sampling prediction horizon for regulation of voltage in system focused on fixed speed generator of disel engine driven. A controller of adaptive PR with scheme of anti-widup is utilized to attain 
regulation of high performance without saturation matters. The adaptive PR controller optimal parameters are obtained by new method to attain high performance through the steady state is given. The current of output filter is managed and controlled by estimated the behavior model of the system at each sampling with step input and the output with performances are verfified by using software in Matlab codes.

Mado, et al., [24], they suggested double seasonal autoregressive integrated moving average (DSARIMA) to estimate load. A method of ccurate fotecasting is desired to retain the reliability and efficiency of power generated system due to variation demand of electric power in the center of electricity load. The DSARIMA model parameters are predicted by method of least square and the result displays the best model for estimate these data.

Hassan, et al., [25], they designed a controller with estimate PI for wireless network. While the model focused conreollers are complecity, the PIDs are provided to handle such challenges. They do not warranty stability under network delay and process variations while measures of classical robustness do not need modeling of extensive uncertainty. The measures of model uncertainty tend to conventional. Consequently, this paper utilitizes method of complementary sensitive function which handle those challenges. The Results displays the controller with PI can be stable under delay and model uncertainties.

Alkurawy and Khamas [26], they designed a mdel predictive controller for Dc motors and did compart the results with proportional integral controller. They linearized the nonlinear system by Tylor method to get the transfer function to do a controller for stability. They got a goor results with low transient response, zero steady state error and no peak overshoot and the results are more sultable than PI controller.

Alkurawy [27], he designed and analyzed a robust control for a nonlinear magnetic levitation system. He linearized a nonlinear syetem and applied robust control to chesck the stability and to included assurance the model uncertainty performance. The results with robust control are very good for stability and zero steady state and minimum peak overshoot and the design is under codes of MATLAB.

\section{RESEARCH METHOD}

Magnetic levitation can be summerly represented as attining the equilibrium of a target in the whole space such that without touch the solid material. A ball is kept in the whole space between two electromagnets by using forces of electromagnetic as shown in Figure 1, where stabilization of a ball horizontal position provided by the lower one and a vertical force overcoming the ball gravity by the upper one.

The physical model of nonlinear model for the magnetic levitation system can be designated by state equations acquired from laws of physical theorems for a ball motion in field of electromagnetic, depiction the dynamics of a ball and the forces of electromagnetic are based on the function of Lagrange and it is the difference between potential and kinetic energy that can be as

$$
T=m g x+q u+\frac{1}{2}\left(\int R\left(\frac{d q}{d t}\right)^{2} d t+L(x)\left(\frac{d q}{d t}\right)^{2}+m x^{\prime^{2}}\right)
$$

where $\mathrm{R}$ is the electromagnet coil resistance, $\mathrm{x}$ is a space of the area from electromagnet, $\mathrm{L}(\mathrm{x})$ is a inductance of the coil on space $\mathrm{x}, \mathrm{g}$ is the constant of a gravity, $\mathrm{q}$ is acharge of electric element, $\mathrm{m}$ is a mass of the area, Variables $\mathrm{x}(\mathrm{t}), \mathrm{q}(\mathrm{t})$ are the equations of the Lagrange $\mathrm{I}=\mathrm{q}^{\prime}$ is the coil current, $\mathrm{u}$ is apply of voltage.

$$
\begin{aligned}
& \frac{\mathrm{d}}{\mathrm{dt}} \frac{\mathrm{dT}}{\left(\frac{d q}{d t}\right)}-\frac{\mathrm{dT}}{\mathrm{dq}}=0 \\
& \frac{\mathrm{d}}{\mathrm{dt}} \frac{\mathrm{dT}}{\left(\frac{d x}{d t}\right)}-\frac{\mathrm{dT}}{\mathrm{dx}}=0 \\
& \frac{\mathrm{dT}}{\left(\frac{d x}{d t}\right)}=\frac{1}{2} \frac{d L}{d x}\left(\frac{d q}{d t}\right)^{2}+m g \\
& \frac{\mathrm{dT}}{\left(\frac{d x}{d t}\right)}=\mathrm{m} \frac{d x}{d t} \\
& \frac{d T}{d t} \frac{\mathrm{dT}}{\left(\frac{d x}{d t}\right)}=m \frac{d^{2} x}{d t^{2}}
\end{aligned}
$$


sub in (2)

$$
\begin{aligned}
& m \frac{d^{2} x}{d t^{2}}-\frac{1}{2} \frac{d L}{d x}\left(\frac{d q}{d t}\right)^{2}-m g=0 \\
& m \frac{d^{2} x}{d t^{2}}=\frac{1}{2} \frac{d L}{d x}\left(\frac{d q}{d t}\right)^{2}+m g \\
& \frac{d^{2} x}{d t^{2}}=\frac{1}{2 m} \frac{d L}{d x}\left(\frac{d I}{d t}\right)^{2}+g \\
& \frac{\mathrm{dT}}{\mathrm{dq}}=u \\
& \frac{d T}{\left(\frac{d q}{d t}\right)}=R q+L(x) \frac{d q}{d t} \\
& \frac{\mathrm{d}}{\mathrm{dt}} \frac{d T}{\left(\frac{d q}{d t}\right)}=R \frac{d q}{d t}+L(x) \frac{d^{2} q}{d t^{2}}+\frac{d q}{d t} \frac{d L}{d x}
\end{aligned}
$$

sub in (2)

$$
\begin{aligned}
& R \frac{d q}{d t}+L(x) \frac{d^{2} q}{d t^{2}}+\frac{d q}{d t} \frac{d L}{d x}-u=0 \\
& L(x) \frac{d^{2} q}{d t^{2}}=-R \frac{d q}{d t}-\frac{d q}{d t} \frac{d L}{d x}+u \\
& I=\frac{d q}{d t} \\
& L \frac{d I}{d t}=-R I+\frac{d L}{d x} \frac{d x}{d t}+u \\
& \frac{d I}{d t}=\frac{1}{L}\left(-R I+\frac{d L}{d x} \frac{d x}{d t}+u\right)
\end{aligned}
$$

By Newton's second law of equation (3), where the force of electromagnetic is described by

$$
F(x, I)=\frac{1}{1} \frac{d L}{d x} I^{2}
$$

The distance of coil inductance can be approached by exponential or polynomial functions

$$
\begin{aligned}
& L(x)=L_{0}+L_{1} e^{-a x} \\
& L(x)=L_{0}+\frac{1}{a_{0}} X^{n}+a_{n-1} X^{n-1}+\cdots+a^{1} X_{1}+a_{0}, n \geq a_{0}, \ldots, a_{n} \in R
\end{aligned}
$$

we will use different the exponential (5a),

$$
\frac{d L}{d x}=-a L_{1} e^{-a x}
$$

By using the second equation of (3) is carified by

$$
\frac{\mathrm{dI}}{\mathrm{dt}}=\frac{1}{\mathrm{f}(\mathrm{x})}(1-\mathrm{c}-\mathrm{ku})
$$

By using the state equation, will get

$$
\begin{aligned}
& x_{1}(t)=x(t) \approx \text { position of the sphere } \\
& x_{2}(t)=x^{\prime}(t) \approx \text { velocity of the sphere } \\
& x_{3}(t)=I(t) \approx \text { current in the upper coil, }
\end{aligned}
$$


By incorporating (3) and (7), a model with nonlinear is got by,

$$
\begin{aligned}
& \frac{d x_{1}}{\mathrm{dt}}=x_{2} \\
& \frac{d x_{2}}{d t}=g+\frac{1}{2 m} \frac{d L\left(x_{1}\right)}{d x_{1}} x_{3}^{2} \\
& \frac{d x_{3}}{d t}=-\frac{1}{f\left(x_{1}\right)}\left(c-x_{3}+k u\right)
\end{aligned}
$$

By approximation (6), we get

$$
\frac{d x_{3}}{d t}=g-\frac{f e m_{1}}{2 m}
$$

where $F e m_{1}=x_{3}^{2} \frac{F e m p_{1}}{F e m p_{2}} \exp \left(-\frac{x_{1}}{F e m p_{2}}\right)$

By equalities from (8) and (9) is through calculation (6) with

$$
\begin{aligned}
& a \approx \frac{1}{F e m p_{2}}, L_{1} \approx F e m p_{1} \\
& \frac{d L}{d x} \approx-\frac{F e m p_{1}}{F e m p_{2}} \exp \left(-\frac{x_{1}}{F e m p_{2}}\right)
\end{aligned}
$$

The linearized state space model for (1) can be obtained using Jacobian linearization around the determined working point as

$$
\begin{aligned}
X^{\prime}(t) & =A x(t)+B u(t) \\
& =\left[\begin{array}{ccc}
0 & 1 & 0 \\
a_{2,1} & 0 & a_{2,3} \\
0 & 0 & a_{3,3}
\end{array}\right] x(t)+\left[\begin{array}{l}
0 \\
0 \\
b_{3}
\end{array}\right] u(t) \\
Y(t) & =C x(t)=\left[\begin{array}{lll}
1 & 0 & 0
\end{array}\right] x(t)
\end{aligned}
$$

When we put voltages on the system, we got the output as an offset in transfer function as in Figure 2.

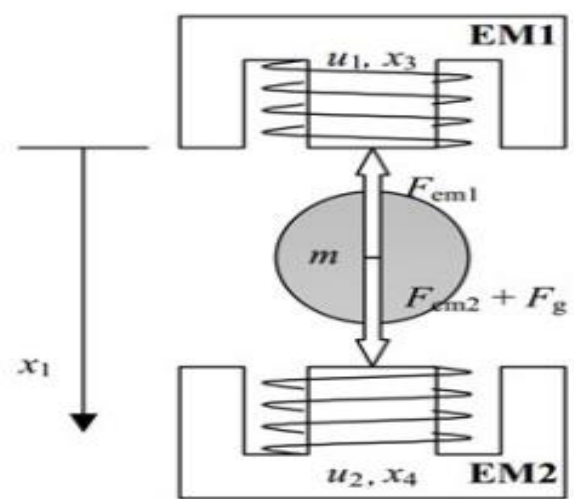

Figure 1. Magnetic levitation system

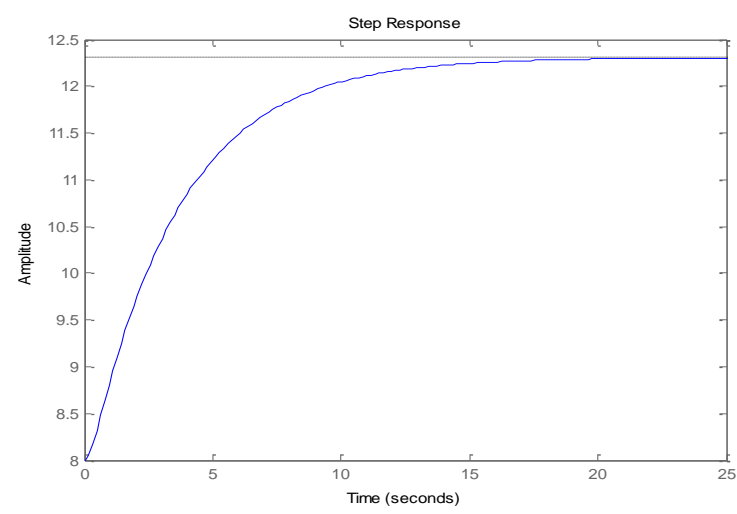

Figure 2. Transfer function of the output when put voltages on the system

\section{PI AND PID CONTROLLER}

The PI controller is the main common controller in the industry over the 60 last years. Though PI controllers can make delays in the dynamic of the systems, in the limitation of single input single output (SISO) controller is approved when the delays are dealing with time-varying. This case can do problem when it delays in values of speed which alters with set points. In order to solve this problem, we investigate the properties of robustness by using PI. The control law of PI controller with Laplace transform is: 


$$
U(s)=\frac{K_{p} S+K_{i}}{s}
$$

The PID controller is the main usual form of closed loop feedback system. It was necessary controller of early regulators to be the essential instrument when the control appeared from the 1950s. The PID controller is a fundamental component for asystem to be stable. The PID controller is established in many purposes control systems. The logic, sequential functions, blocks of simple function, and selectors used with PID to design a system if the complicated automation that used for manufacturing energy production, and transportation. The control law of PI control as the Laplace transform is:

$$
U(s)=\frac{K_{d} s^{2}+K_{p} s+K_{i}}{s} e(s)
$$

\section{MODEL PREDICTIVE CONTROL FOR MAGNETIC LEVITATION SYSTEM}

Model Predictive Control (MPC) has been used in the family of control engineering. The researchers of academic and industrial communities have been used the area of receirved on-going interest. There are three steps of model predictive control to make the design both academics and engineering. The first step is the structure expression, which utilizes a totally multivariable system structure where the parameters of performance of the multivariable control system are associated to the features of engineering for the system; consequently, they can be accepted by engineers. The second step is with hard condtfraints and soft constraints in a framework of multivariable control. This is specially appeling to manufacturing where tight advantage margins and boundaries on the operation of process. The third step is the capability for optimization to excute process on-line.

In the generality of applications, the model predictive control is structured to follow reject disturbances and signals of constant reference that have mostly low frequency contents. Nevertheless, these kinds of MPC are not sufficient to supply the performance with closed-loop required when the disturbances and signals of set-point have periodic elements. This has derived the speakers to establish a new class of MPC that use the design of MPC framework while the controlling system with components of periodic for addressing, that named predictive repetitive controller as shown in Figure 3.

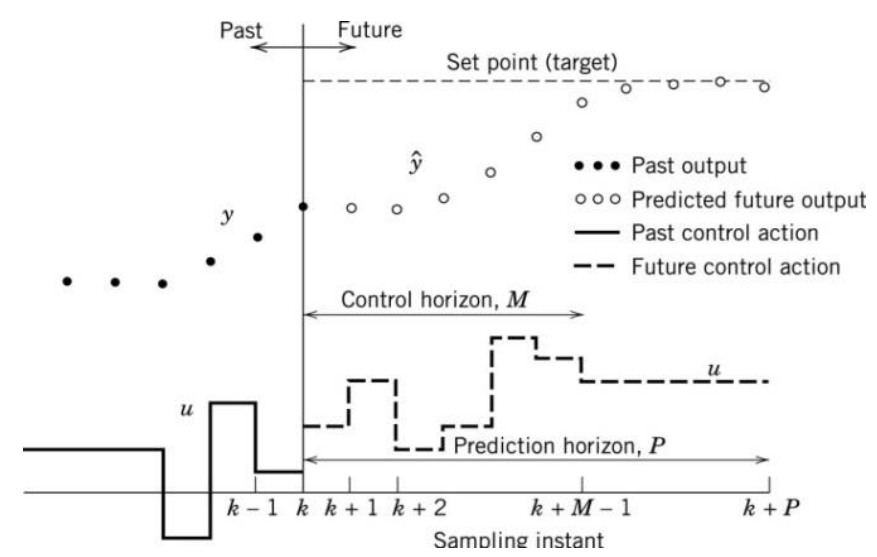

Figure 3. Basic concept for model predictive control

All the algorithms of model predictive control own different choices and ordinary parts can be selected for each one of these parts giving rise to different algorithms. Three elements for MPC; prediction model, objective function and control law. To determine the predicted output at future instants by using the process of the process that is calculated by the necessity

$$
\hat{\mathrm{y}}(t+k)
$$

where $\hat{y}$ is a p-dimensional vector containing the system prediction along the horizon.

The requirements of plant operation calculate the control system in the objective function. These criteria have to be calculated in terms of mathematical way so that a control law can be got in algorithmic pattern. A function of quadratic objective is: 


$$
\min _{\Delta u(k) \ldots \ldots . \Delta \mathrm{u}(\mathrm{k}+\mathrm{m}-1)} \sum_{l=1}^{p}\|y([y(k+l \mid k)-r(k+l)])\|^{2}
$$

This can be influenced transparently by including weights in the objective function as follows:

$$
\min _{\Delta u(k)} \sum_{l=1}^{p}\left\|\Gamma_{l}^{y}[([y(k+l \mid k)-r(k+l)])]\right\|^{2}+\sum_{l=1}^{m} \| \Gamma_{l}^{u}\left[\Delta_{u}(k+l-1] \|^{2}\right.
$$

where $\Gamma_{l}^{y}$ and $\Gamma_{l}^{u}$ are matrices of weighting to penalize spesific components of $y$ or $u$ at $r(k+1)$ as set point for future time. The moving horizon is leading to get the control law, the feedback control law is

$$
\Delta u(k)=K_{M P C} E_{p}(k+1 \mid k)
$$

where $E_{p}(k+1 \mid k)$ is error of the predicted future over the horizon $P$ which would result if all variable of future and present manipulated moves were equal to zero $\Delta u(k)=\Delta u(k+1)=\cdots=0$. The problem of optimization with linear inequalities and quadratic objective, which it has determined is a Quadratic Program $(\mathrm{QP})$. The $\mathrm{DMC}$ problem becomes:

$$
\min _{\Delta u(k)} \Delta u(k)^{T} H^{u} \Delta u(k)-g(k+1)^{T} \Delta u(k)
$$

\section{RESULTS AND ANALYSIS}

For testing the response of magnetic levitation system in closed loop with PI and PID controller by changing the values of $K_{p}, K_{i}$ and $K_{d}$ and will see some of figures have peak overshoot, large steady state error and large settling time and make these controllers are uncomfortable for this system as shown in Figures 4 to 10 .

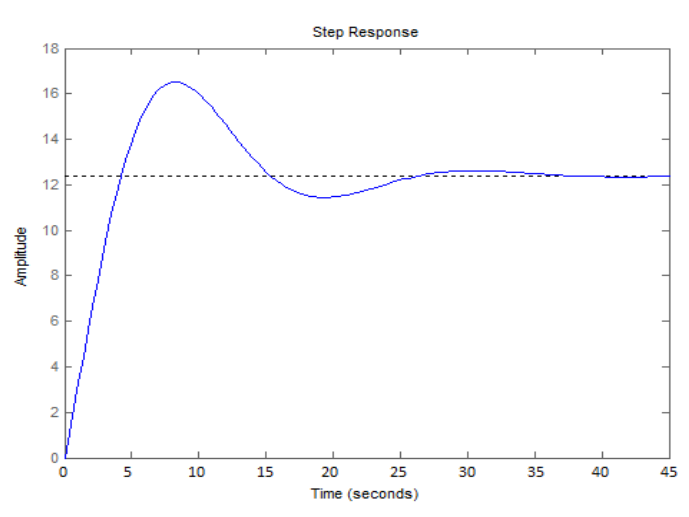

Figure 4. The amplitude with $\mathrm{Kp}=1, \mathrm{Ki}=10$

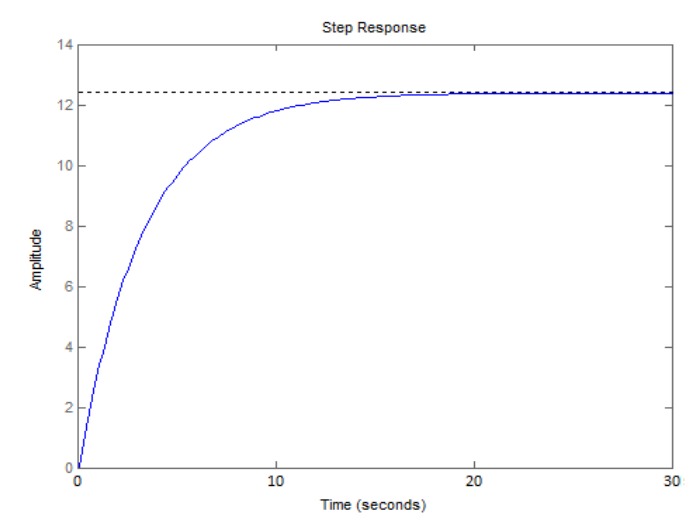

Figure 6. The amplitude with $\mathrm{Kp}=10, \mathrm{Ki}=0.1$

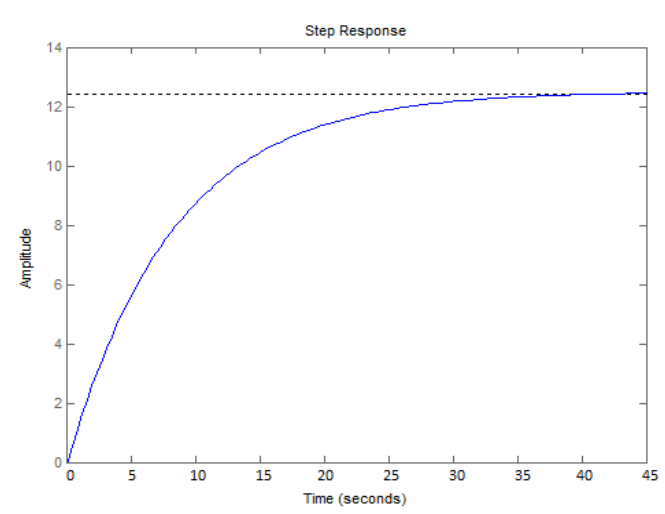

Figure 5. The amplitude with $\mathrm{Kp}=10, \mathrm{Ki}=10$

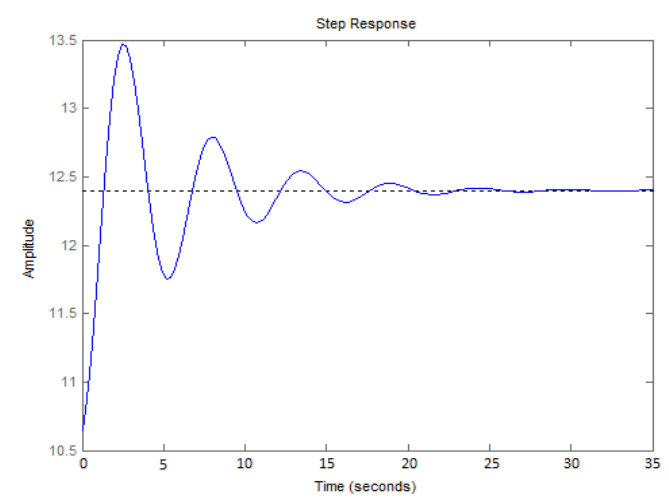

Figure 7. The amplitude with $\mathrm{Kp}=1$, $\mathrm{Ki}=10, K_{d}=1$ 


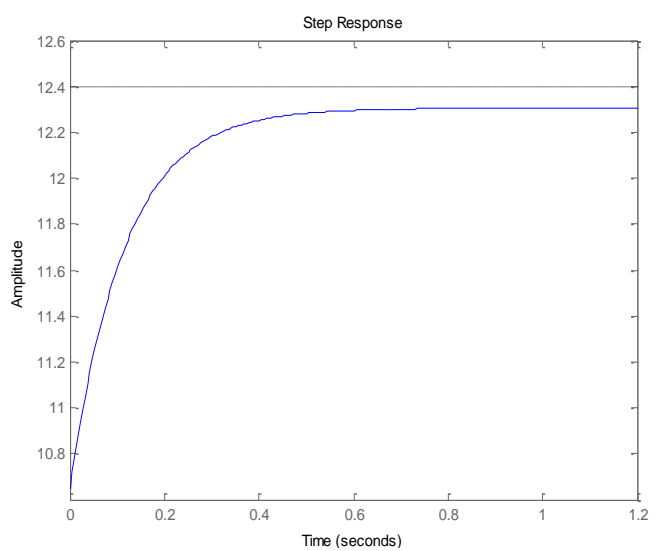

Figure 8. The amplitude with $\mathrm{Kp}=10, \mathrm{Ki}=0.10, K_{d}=10$

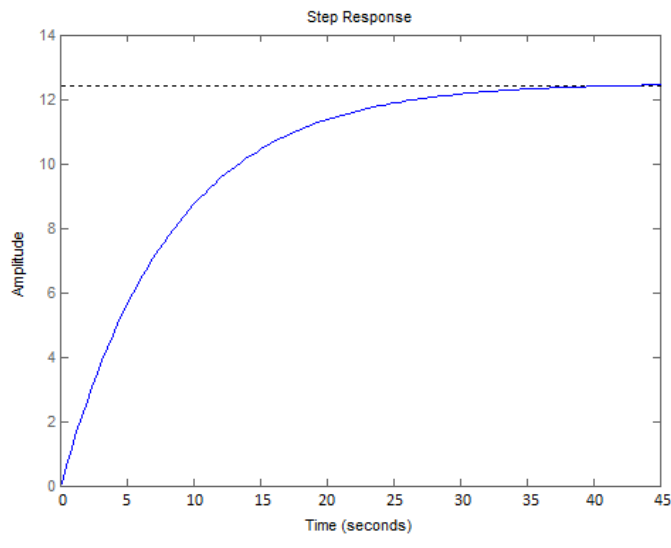

Figure 9. The amplitude with $\mathrm{Kp}=10, \mathrm{Ki}=10, K_{d}=0.01$

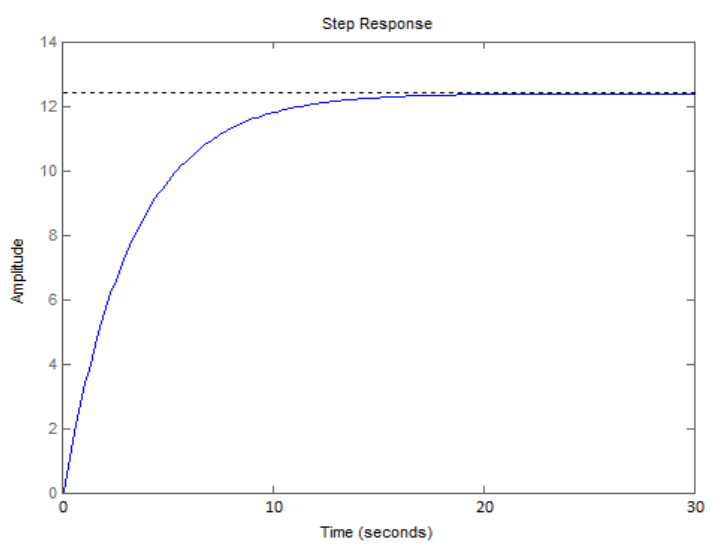

Figure 10. The amplitude with $\mathrm{Kp}=100, \mathrm{Ki}=1, K_{d}=0.001$

For testing the response the magnetic levitation system by model predictive control by changing the values of weighting matrices $\Gamma_{y}$ and $\Gamma_{u}$ and will see all responses are comfortable with this controller because the peak overshoot disappeared, the steady state error is zero and minimum settling time and that refers to get fast response. The choosing values of $\Gamma_{y}$ and $\Gamma_{u}$ are tuning to get good response without peak overshoot, minimum settling time and zero steay state error. The best values of $\Gamma_{y}$ and $\Gamma_{u}$ for the system when they have large values as shown in Figures 11-13.

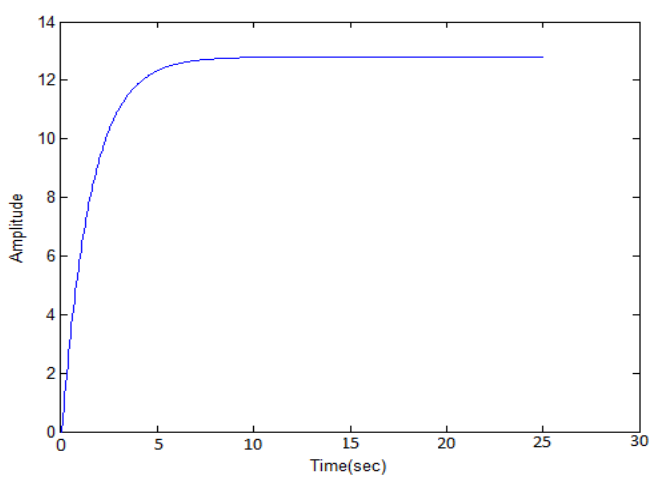

Figure 11. The amplitude at $\Gamma_{y}=1, \Gamma_{u}=0$

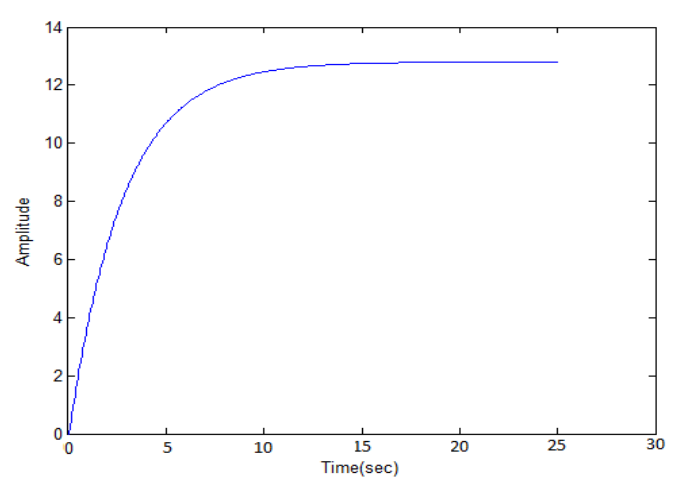

Figure 12. The amplitude at $\Gamma_{y}=1, \Gamma_{u}=5$ 


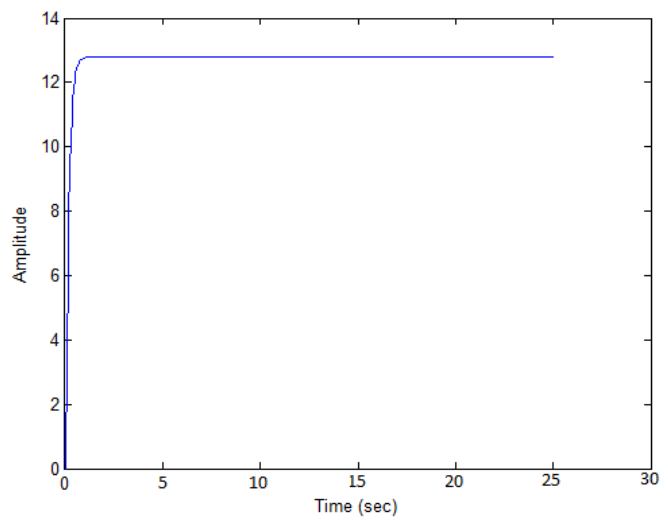

Figure 13. The amplitude at $\Gamma_{y}=2, \Gamma_{u}=10$

\section{CONCLUSION}

The work applied in this paper was designed the dynamic magnetic levitation of nonlinear system that was investigated in terms of its modeling, simulation and linearization. Because of the non-linear and unstable and featur of magnetic levitation systems, in this paper, we tried to convert the nonlinear to the linearize model by analyzing with state-space and doing a simulation. Three controllers applied to the system to check what it is the best controller. The first and second classic control were PI controller and PID controller and these controllers were not satisfied for stability and steady state error. The third controller was MPC to predict the future response related to reference input and this controller was satisfied for stability, get zero steady state error and fast response with minimum settling time. Further, a new research uses linear quadratic form to control the magnetic levitation syatem to ensure the stability with changing the input.

\section{REFERENCES}

[1] H. Seo, et al., "Algorithm of Linear Induction Motor Control for Low Normal Force of Magnetic Levitation Train Propulsion System," IEEE Transactions on Magnetics, vol. 54, no. 11, pp. 1-4, 2018.

[2] T. Takao, et al., "Improvement of Levitation Properties owing to High Magnetic Field in a Magnetic Levitation System with Magnetic Shielding Effect of HTS Bulk," IEEE Transaction on Applied Superconductivity, vol. 28, no. 4, pp. 1-5, 2018.

[3] R. Sun, et al., "Dynamic Characteristics of the Manned Hybrid Maglev Vehicle Employing Permanent Magnetic Levitation (PML) and Superconducting Magnetic Levitation (SML)," IEEE Transaction on Applied Superconductivity, vol. 29, no. 3, pp. 1-5, 2019.

[4] J. Lim, et al., "A Study on Optimal Operating Point of Linear Induction Motor Considering Normal Force and Efficiency in MAGLEV Vehicle," IEEE Transaction on Magnetics, vol. 54, no. 11, pp. 1-5, 2018.

[5] W. Chen, et al., "Levitation Force Computation of HTS/PM System Based on H-Formulation," IEEE Transaction on Magnetics, vol. 54, no. 11, pp. 1-5, 2018.

[6] F. Xu, et al., "FPGA-Based Real-time Wrench Model of Direct Current Driven Magnetic Levitation Actuator," IEEE Transaction on Industrial Electronics, vol. 65, no. 12, pp. 9635-9645, 2018.

[7] M. Y. Chen, et al., "A Novel Design and Control to Improve Positioning Precision and Robustness for a Planar Maglev System," IEEE Transactions on Industrial Electronics, vol. 66, no. 6, pp. 4860-4869, 2019.

[8] D. Spałek, "Levitation of Conductive and Magnetically Anisotropic Ball," IEEE Transaction on Magnetics, vol. 55, no. 3, pp. 1-7, 2019.

[9] A. Morandi, et al., "The Measurement and Modeling of the Levitation Force between Single-Grain YBCO Bulk Superconductors and Permanent Magnets," IEEE Transactions on Applied Superconductivity, vol. 28, no. 5, pp. 1-10, Aug. 2018.

[10] Y. Chen, et al., "Numerical Analysis and Experimental Study on Magnetic Fluid Reciprocating Seals," IEEE Transactions on Magnetic, vol. 55, no. 1, pp. 1-6, Jan. 2019.

[11] L. Qi, et al., "A novel nonlinear control technique with its application to magnetic levitated systems," IEEE Access, vol. 6, pp. 78659-78665, 2018.

[12] M. Lahdo, et al., "Repulsive Magnetic Levitation Force Calculation for a High Precision 6-DoF Magnetic Levitation Positioning System," IEEE Transactions on Magnetics, vol. 53, no. 3, pp. 1-6, 2017.

[13] L. E. Venghi, et al., "Implementation and Control of a Magnetic Levitation System," IEEE Latin America Transactions, vol. 14, no. 6, pp. 2651-2656, Jun. 2016.

[14] S. Sivrioglu, et al., "Multi surface HTS-PM levitation for a flywheel system," IEEE Transaction on Applied Superconductivity, vol. 26, no. 8, pp. 1-6, 2016. 
[15] A. Patel, et al., "Magnetic Levitation between a slab of soldered HTS Tape and a Cylindrical Permanent Magnet," IEEE Transaction on Applied Superconductivity, vol. 26, no. 3, pp. 1-5, 2016.

[16] W. Amrhein, et al., "Magnetic Levitation Systems for Cost-Sensitive Applications-Some Design Aspects," IEEE Transaction on Industry Applications, vol. 52, no. 5, pp. 3739-3752, 2016.

[17] S. F. AL-Azzawi, an dM. M. Aziz, "Strategies of linear feedback control and its classification," TELKOMNIKA (Telecommunication, Computing, Electronics and Control), vol. 17, no. 4, pp. 1931-1940, Aug. 2019.

[18] S. F. Sulaiman, M. F. Rahmat, A. A. M. Faudzi, and K. Osman, "A new technique to reduce overshoot in pneumatic positioning system," TELKOMNIKA (Telecommunication, Computing, Electronics and Control), vol. 17, no. 5, pp. 2607-2616, Oct. 2019.

[19] N. H. Quang, et al., "Multi parametric model predictive control based on laguerre model for permanent magnet linear synchronous motors," International Journal of Electrical and Computer Engineering (IJECE), vol. 9, no. 2, pp. 1067-1077, Apr. 2019.

[20] O. Kennedy, et al., "Comparative analysis of the performance of vrious active queue management techniques to varying wireless network conditions," International Journal of Electrical and Computer Engineering (IJECE), vol. 9, no. 1, pp. 359-368, Feb. 2019.

[21] S. Firdose and L. M. Rao, "PORM: Predictive Optimization of Risk Management to Control Uncertainty Problems in Software Engineering," International Journal of Electrical and Computer Engineering (IJECE), vol. 8, no. 6, pp. 4735-4744, Dec. 2018.

[22] S. A. Yasear, K. R. Ku-Mahamud, "Non-dominated sorting Harris's hawk multi-objective optimizer based on reference point approach," Indonesian Journal of Electrical Engineering and Computer Science, vol. 15, no. 3, pp. 1603-1614, Sep. 2019.

[23] M. B. Shahid, M. A. Abbasi, and S. Arsha, "Asymptotic features of Hessian Matrix in Receding Horizon Model Predictive Control with Medium Sized Prediction Frames," TELKOMNIKA (Telecommunication, Computing, Electronics and Control), vol. 16, no. 3, pp. 1174-1182, Jun. 2018.

[24] I. Mado, et al., "Applying of Double Seasonal ARIMA Model for Electrical Power Demand Forecasting at PT.PLN Gresik Indoensia," International Journal of Electrical and Computer Engineering (IJECE), vol. 8, no. 6, pp. 48924901, Dec. 2018.

[25] S. M. Hassan, et al., "Robustness and Stabilty Analysis of a Predictive PI Controller in WirelessHart Network Characterised by Stochastic Delay," International Journal of Electrical and Computer Engineering (IJECE), vol. 7, no. 5, pp. 2605-2613, Oct. 2017.

[26] L. E. J. Alkurawy and N. Khamas, "Model Predictive Control for DC Motors," 1st International Scientific Conference of Engineering Sciences-3rd Scientific Conference of Engineering Science (ISCES), pp. 56-61, 2018.

[27] L. E. J. Alkurawy, "Simulation of Robust Control of Magnetic Levitation System," Journal of Engineering and Applied Sciences, vol. 14, no. 13, pp. 4523-4531, 2019.

\section{BIOGRAPHIES OF AUTHORS}

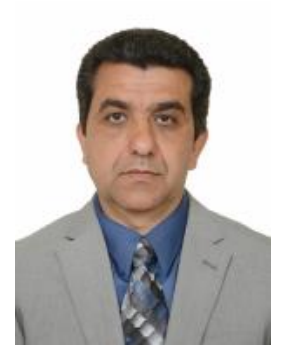

Assist Prof. Lafta E. Jumaa Alkurawy received the B.S., and M.S. degree in Control and systems from Technology University, Baghdad, Iraq, in 1990 and 2003 respectively. He received the Ph.D. degree in Electrical and Computer Engineering from the University of Missouri in Columbia, USA, in 2013. Since 2003, I have been with the University of Diyala, College of engineering, Diyala, Iraq as a lecturer. His current research interests include modeling, control, Numerical analysis and nonlinear.

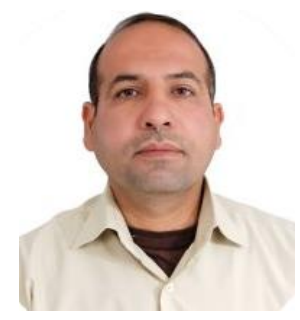

Assist Prof. Khalid G. Mohammed is a PhD researcher in the Department of Electrical Power Engineering at University Tenaga Nasional, Malaysia. He has ten years of industrial experience in repairing and maintenance of several types of electrical machines. He has published many papers works in 2007 and 2019 containing many new practical correlations for the design of single and three phase induction motors. He has authored three practical experimentation books in electrical circuits and machines and has been teaching since 2009 in the College of Engineering, Diyala University, Iraq. 